\title{
PROTECTIVE ROLE OF AQUEOUS EXTRACT OF ATRIPLEX HALIMUS L. AGAINST BENZENE-INDUCED DAMAGE ON RENAL FUNCTION AND GLOMERULAR CELLS IN RATS
}

\author{
KHAOULA ZEGHIB ${ }^{1,2 *}$, DJAHRA ALI BOUTLELIS ${ }^{3}$ \\ ${ }^{1}$ Department of Chemistry, Faculty of exact sciences, University of El-Oued, El-Oued 39000, El-Oued, Algeria. ${ }^{2}$ University of El Oued, VTRS \\ Laboratory, Fac. Technology, 39000 El Oued, Algeria. ${ }^{3}$ Department of Biology, Faculty of Natural Sciences and Life, University of El-Oued, \\ El-Oued 39000, Algeria. Email: zeghib-khaoula@univ-eloued.dz
}

Received: 13 November 2018, Revised and Accepted: 01 January 2019

ABSTRACT

Objective: Benzene (BZ) is an important pollutant compound, present in both occupational and general environment that has been linked to adverse health effect. The aim of the current study was to investigate the curative and preventive effect of Atriplex halimus L. (Ah) extract against BZ-induced nephrotoxicity in rats.

Methods: A total of 30 male albino Wistar rats were divided into five groups (n=6): Control, Ah, BZ, AhP+BZ, and BZ+AhC. BZ (100 mg/kg b.w) was added in their drinking water for 15 weeks. Ah (Aqueous Extract of aerial parts) was given by gavage during the past 30 days of BZ-exposed for curative treatment (AhC) and all the duration of BZ exposure in the animals for preventive treatment (AhP). Some biochemical, oxidative stress parameters, and histopathology of kidney tissue were studied.

Results: Obtained results revealed that BZ exposure to rats caused a significant elevation in urea, creatinine, and malondialdehyde levels. Then, it led to reducing the glutathione level, catalase, and glutathione-S-transferase activities when compared to the control animals. Histopathological studies showed a massive damage in kidneys of the BZ-exposed rat. Our results showed that curative treatment of aqueous extract of Ah has a partial correction of the biochemical, oxidative stress parameters, and kidney morphology, but preventive treatment has a dramatic effect on the previous parameters.

Conclusion: Results demonstrated the beneficial effects of Ah preventive treatment against stress oxidative and kidney damage induced by BZ.

Keywords: Atriplex halimus, Benzene, Oxidative stress, Nephrotoxicity.

(C) 2019 The Authors. Published by Innovare Academic Sciences Pvt Ltd. This is an open access article under the CC BY license (http://creativecommons. org/licenses/by/4. 0/) DOI: http://dx.doi.org/10.22159/ajpcr.2019.v12i3.30741

\section{INTRODUCTION}

Benzene (BZ) with C6H6 formula is the primary aromatic compound [1]. It has been extensively used since 100 years and is still widely used as a solvent and intermediate compound [2] and produced in large quantities in the chemical and fuel industry including gasoline, automobile exhaust, several polymers, resins, synthetic fibers, and cigarette smoke [3]. It is also used in manufacturing products such as rubber, detergents, lubricants, pesticides, and drugs [4]. Workers employed in industries that manufacture or use BZ is exposed to its highest levels [5]. BZ is a ubiquitous environmental pollutant [6] which affects human health includes hematological diseases, reproductive system, respiratory problems, kidney disease, liver injury, and central nervous system [7]. Thus, humans are unavoidably exposed to BZ both occupational and environmental settings and which poses a serious risk to public health [8].

Toxicity of BZ most likely results from oxidative metabolism of BZ to reactive products [9]. These reactive products can lead to the formation of reactive oxygen species (ROS), which can directly damage pivotal molecular targets, such as DNA, proteins, and lipids, leading to the formation of new compounds and modified structures [10], and in this condition; oxidative stress has a critical role in the pathophysiology of several kidney diseases $[11,12]$.

The association between food and health is well established. Recent overwhelming attention to plant products and traditional medicine has focused on natural antioxidant for preventing the tissues from various xenobiotics induced toxicities [13,14]. Atriplex halimus L. (Ah) is a famous halophytic shrub for the nutritive and energetic value, not only for livestock but also as food for nomads and the local steppe population.
The young shoots of Guettaf are eaten by the man, preparing its like spinach [15]. Ah also has always been a popular folk remedy for treating hyperglycemia, stomach painkiller, and intestinal worms [16]. In light, these considerations, the aim of the present study is to determine the effects of Ah against BZ-induced nephrotoxicity in rats.

\section{METHODS}

Chemicals and reagents

All chemicals used in this study were purchased from Sigma-Aldrich, Mo, USA.

\section{Plant material}

Aerial parts of Ah were collected in September 2017 from a village in Sidi Khaled of Biskra state, Algeria and were identified by a botanist (Helios Youssef) at the herbarium in the Department of Biology, the University of El-Oued, Algeria. The plant material was washed using water, dried at room temperature, and grounded into powder and then stored at room temperature until use.

Preparation of aqueous extract

About $10 \mathrm{~g}$ of the aerial parts powder of Ah was soaked in $100 \mathrm{ml}$ of distilled water and kept at room temperature in the dark for $24 \mathrm{~h}$. It was filtered through Whatman filter paper. After extraction, the solvent was removed using a rotary evaporator and incubated at $40^{\circ} \mathrm{C}$ to dry completely. The extract was weighed and stored in a refrigerator at $4^{\circ} \mathrm{C}$ for future analysis [17].

\section{Estimation of total phenolic}

Total phenolic content was estimated by the Folin-Ciocalteu method. This method initially described by Singleton and Rossi (1965). The 
sample of the aqueous extract of $\mathrm{Ah}(0.2 \mathrm{ml})$ and $1 \mathrm{ml}$ of $1: 10$ diluted Folin-Ciocalteu reagent were added after $4 \mathrm{~min}$ to $800 \mu \mathrm{l}$ of saturated sodium carbonate $(75 \mathrm{~g} / \mathrm{l})$. After $2 \mathrm{~h}$ of incubation at room temperature, the absorbance was measured at $765 \mathrm{~nm}$. The tests were carried out 3 times to ensure the reproducibility of the results [18]. The total phenolic content was expressed in mg equivalent of gallic acid per gram of sample.

\section{Estimation of condensed tannin}

The tannins content in the extract was determined spectrophotometrically according to Broadhurst and Jones [19]. Catechin was used to make the calibration curve. $0.5 \mathrm{ml}$ of sample pipetted into a tube in aluminum foil, and mixed with $3.0 \mathrm{ml}$ of vanillin reagent (Freshly prepared $4 \% \mathrm{w} / \mathrm{v}$ of vanillin in methanol), and then added $1.5 \mathrm{ml}$ of concentrated hydrochloric acid and mixed thoroughly. After $15 \mathrm{~min}$ of reaction at $20-2^{\circ} \mathrm{C}$, the absorbance was measured against water at $500 \mathrm{~nm}$.

\section{In vivo study}

\section{Animals and treatment}

A total of 30 adult male albino rats, weighing $227.14 \pm 3.42 \mathrm{~g}$, were taken from the animal house of Pasteur Institute, Algeria. They were placed in five groups of six rats in each and kept in animal's house of Molecular and Cellular Biology Department, University of El-Oued, Algeria. Standard rat food and tap water were available ad libitum for the duration of the experiments. Animals were adapted for 2 weeks under the same laboratory conditions of photoperiod (12 h light/12 h dark) with relative humidity $62 \%$ and room temperature of $24 \pm 1^{\circ} \mathrm{C}$. The experimental procedures were carried out according to the National Institute of Health Guidelines for Animal Care and approved by the Ethics Committee of our Institution. The adult Wistar albino rats were randomly divided into five groups, each containing 6 rats as follow:

- Group 1 was orally received normal water and serving as a control.

- Group 2 was received oral gavage $200 \mathrm{mg} / \mathrm{kg}$ b.w (3 days/week) aqueous extracts of Ah for 15 weeks.

- Group 3 treated by $100 \mathrm{mg} / \mathrm{kg}$, bw/day of BZ (Fig. 1) for 15 weeks.

- Group 4 was first treated by BZ only for 11 weeks then treated curatively by $300 \mathrm{mg} / \mathrm{kg}$, bw/day of $\mathrm{Ah}(\mathrm{BZ}+\mathrm{AhC})$ for 30 days.

- Group 5 was concomitantly administered preventively Ah (200 mg/ kg, 3 days/week) with BZ for 15 weeks (AhP+BZ).

During treatment, body weight was recorded periodically during the experiment weeks.

\section{Blood collection and preparation of tissue samples}

At the end of treatment, rats were fasted for $16 \mathrm{~h}$, anesthetized with chloroform by inhalation, rats were decapitated, and blood samples were transferred into ice-cold centrifuge tubes. The serum was prepared by centrifugation for $10 \mathrm{~min}$ at 3000 revolutions/min and utilized for urea, creatinine, albumin, uric acid, and total proteins concentrations, and lactate dehydrogenase (LDH) activity assays. Blood glucose was measured by glucometer. The kidney was rapidly excised, washed, weighed, and stored at $-20^{\circ} \mathrm{C}$ for oxidative stress parameters analysis. Another part of the kidney was excised for histopathological examination.

\section{Measurement of biochemical parameters}

Serum urea, creatinine, albumin, uric acid, and total proteins concentrations, and LDH activity were measured using commercial kits obtained from Spinreact (Barcelona, Spain).

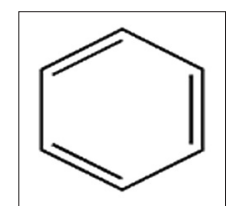

Fig. 1: Chemical structure of benzene [20]

\section{Antioxidants measurement}

\section{Preparation of homogenates}

About $1 \mathrm{~g}$ of the kidney was homogenized in $9 \mathrm{ml}$ of buffer solution of Tris buffer saline (TBS, $\mathrm{pH}=7.4$ ). Homogenates were centrifuged at $9000 \times \mathrm{g}$ for $30 \mathrm{~min}$ at $4^{\circ} \mathrm{C}$, and the obtained supernatant was used for the determination of oxidative stress markers.

\section{Determination of malondialdehyde (MDA) level}

MDA was measured according to the method described by Quintanilha et al. [21]. The MDA reagent (trichloroacetic acid, thiobarbituric acid, and hydrochloric acid) was added to aliquots of the homogenate mixed with $2 \%(\mathrm{w} / \mathrm{v})$ ethanolic solution of butylated hydroxytoluene. Then, the mixture was heated for $15 \mathrm{~min}$ in a boiling water bath. After cooling, the precipitate was removed by centrifugation, and the absorbance was measured at $532 \mathrm{~nm}$. The results were expressed as nmol of MDA/mg protein.

\section{Determination of reduced glutathione (GSH) level}

GSH concentration was performed with the method described by Ellman (1959) based on the development of yellow color when DTNB is added to compounds containing sulfhydryl groups. In brief, $0.8 \mathrm{ml}$ of tissue homogenate was added to $0.2 \mathrm{ml}$ of $0.25 \%$ sulfosalicylic acid and tubes were centrifuged at $2500 \mathrm{~g}$ for $15 \mathrm{~min}$. Supernatant $(0.5 \mathrm{ml})$ was mixed with $0.025 \mathrm{ml}$ of $0.01 \mathrm{M}$ DTNB and $1 \mathrm{ml}$ TBS (pH 7.4). Finally, the absorbance at $412 \mathrm{~nm}$ was recorded. Total GSH content was expressed as nmol GSH/g tissue [22].

\section{Determination of glutathione-S-transferase (GST) activity}

GST activity of tissues was measured spectrophotometrically by the method of Habig et al. [23]. Using CDNB as an electrophilic substrate that binds to GSH with the participation of the enzyme and forms a colored GSH-substrate complex, detected at $340 \mathrm{~nm}$. The activity of GST was expressed in terms of $\mu \mathrm{mol}$ CDNB-GSH conjugate formed $/ \mathrm{min} / \mathrm{g}$ tissue.

\section{Determination of catalase (CAT) activity}

CAT activity was assayed by the decomposition of hydrogen peroxide according to Aebi method, and the absorbance of the sample was measured at $240 \mathrm{~nm}$ for $1 \mathrm{~min}$ using a UV-spectrophotometer. The enzymatic activity was calculated in terms of the international unit/min/g of tissue [24].

\section{Protein level assay}

The protein content of the supernatant was spectrophotometrically estimated by Bradford method using bovine serum albumin as standard [25].

\section{Histopathological study}

After rats sacrificed, the part of kidney tissues was removed and immersed in fixative (solution 10\% formaldehyde) until the time of slices preparation. It was dehydrated in ascending graded series of ethanol, cleaned with toluene, and immersed in paraffin. Sections of 4 6 Mm were prepared from paraffin blocks using a Leica Rotary Microtome (Wetzlar, Germany), and colored with hematoxylin and eosin. Histopathological evaluation was performed with a light microscope.

\section{Statistical analysis}

Data were carried out using Student's t-test to compare means among the groups. Differences were considered statically significant at $p<0.05$.

\section{RESULTS}

Phenolic and tannin compounds

Total phenolic and condensed tannin compounds were expressed in terms of gallic acid equivalents (mg of GAE/g sample) and of catechin equivalents (mg of $\mathrm{Ca} / \mathrm{g}$ sample), respectively, using the 
following equation based on the calibration curve: $Y=0.0104 x+0.0819$, $\mathrm{R} 2=0.9925$ for phenolic compounds and $\mathrm{Y}=0.003 \mathrm{x}+0.006 \mathrm{R} 2=0.9882$ for condensed tannin compounds. Total phenolic and condensed tannin contents of aqueous Ah extract were represented in Table 1.

\section{Body and relative kidney weight}

BZ exposure rats caused a decrease in body weight gain $(p<0.001)$ and an increase in relative kidney weight $(\mathrm{p}<0.01)$ compared to the control rats. Preventive Ah treatment of $\mathrm{BZ}$ exposure rats $(\mathrm{AhP}+\mathrm{BZ})$ was totally restored the body weight gain $(\mathrm{p}<0.05)$ and relative kidney weight $(\mathrm{p}<0.05)$ level as compared to the BZ rats. Curative treatment of the extract aqueous of Ah was significantly $(\mathrm{p}<0.05)$ increase body weight gain compared to $\mathrm{BZ}$ rats, but no effect for on relative kidney weight in

Table 1: Total phenolic and tannin content

\begin{tabular}{ll}
\hline Compounds & Atriplex halimus \\
\hline Total phenolic (mg GA eq/g dry wt) & $17.183 \pm 0.067$ \\
Condensed tannin (mg Ca eq/g dry wt) & $1.49 \pm 0.020$ \\
\hline Data are expressed as mean \pm SE; $n=3$. GA: Gallic acid, Ca: Catechin, SE: Standard \\
error
\end{tabular}

$(\mathrm{BZ}+\mathrm{AhC})$ rats compared to $\mathrm{BZ}$ rats. No effect on body weight gain and relative kidney weight of $\mathrm{Ah}$ alone treatment in rats compared to the control rats (Table 2).

\section{Blood biochemical values}

As shown in Tables 3 and 4, BZ exposure caused a significant increase $(p<0.05)$ in serum creatinine and urea level and a significant decrease in serum protein $(p<0.01)$ levels. Furthermore, no effect of Ah alone or BZ on blood glucose, blood ratio urea/creatinine, serum albumin concentration and LDH activity compared to control. Meanwhile, Ah made a recovery in the above-mentioned biochemical parameters for preventive treatment $(\mathrm{AhP}+\mathrm{BZ})$ but the low effect for curative treatment $(\mathrm{BZ}+\mathrm{AhC})$. In another way, treatment with the aqueous extract of Ah alone has no effect on most of these parameters.

\section{Oxidative stress parameters}

Results presented in Table 5 show a significant increase $(\mathrm{p}<0.05)$ in MDA level and a significant decrease in GSH concentration $(\mathrm{p}<0.01)$, GST $(p<0.01)$, and CAT $(p<0.001)$ activities in BZ group compared to the corresponding control values. Treatment with Ah partially restored the MDA levels $(\mathrm{p}<0.001)$, CAT $(\mathrm{p}<0.05)$, and GST $(\mathrm{p}<0.05)$ activities

Table 2: Initial body weight, body weight gain, and relative kidney weight in control and experimental groups

\begin{tabular}{lllll}
\hline Parameters & Initial body weight $(\mathbf{g})$ & Body weight gain (g/d) & Absolute kidney weight (g) & Relative kidney weight (g/100 g bw) \\
\hline Control & $221.17 \pm 7.73$ & $0.74 \pm 0.10$ & $1.35 \pm 0.031$ & $0.479 \pm 0.019$ \\
$\mathrm{Ah}$ & $233 \pm 4.71$ & $0.719 \pm 0.064$ & $1.49 \pm 0.051$ & $0.48 \pm 0.011$ \\
$\mathrm{BZ}$ & $234.2+12.1$ & $0.38+0.031^{* * *}$ & $1.39+0.072$ & $0.51+0.007^{* *}$ \\
$\mathrm{BZ}+\mathrm{AhC}$ & $229.25+6.34$ & $0.66+0.037^{\Phi \Phi}$ & $1.43+0.065$ & $0.49+0.01$ \\
$\mathrm{AhP}+\mathrm{BZ}$ & $219.25+7.16$ & $0.76+0.072^{\Phi \Phi}$ & $1.39+0.065$ & $0.475+0.01^{\Phi \Phi}$ \\
\hline
\end{tabular}

Data are expressed as mean $\pm \mathrm{SE} ; \mathrm{n}=6$. For Student's t-test significant $\mathrm{p}$ value $\mathrm{Ah}, \mathrm{BZ}, \mathrm{BZ}+\mathrm{AhC}$, and $\mathrm{AhP}+\mathrm{BZ}$ versus control ${ }^{* *} \mathrm{p} \leq 0.01,{ }^{* * *} \mathrm{p} \leq 0.001$ and therapy versus $\mathrm{BZ}$ $\Phi \Phi \mathrm{p} \leq 0.01$. Ah: Atriplex halimus, AhC: Atriplex halimus curative treatment, AhP: Atriplex halimus Preventive treatment, BZ: Benzene, SE: Standard error

Table 3: Mean blood glucose levels and blood biochemical value in the control and experimental groups

\begin{tabular}{lllll}
\hline Parameters & Blood glucose $(\mathrm{g} / \mathbf{l})$ & Serum urea $(\mathrm{g} / \mathbf{l})$ & Serum creatinine $(\mathbf{m g} / \mathbf{l})$ & Blood ratio urea/creatinine \\
\hline Control & $1.026 \pm 0.059$ & $0.27 \pm 0.017$ & $7.45 \pm 0.16$ & $37.58 \pm 3.85$ \\
Ah & $0.93 \pm 0.039$ & $0.28 \pm 0.009$ & $7.85 \pm 0.20$ & $35.66 \pm 2.84$ \\
BZ & $1.038+0.09$ & $0.337+0.015^{* *}$ & $37.41+3.94$ \\
BZ+AhC & $1.05+0.028$ & $0.33+0.02^{*}$ & $8.20+0.34$ & $40.24+5.88$ \\
AhP+BZ & $0.82+0.19$ & $0.24+0.016^{\Phi \Phi}$ & $7.65+0.22^{\Phi \Phi}$ & $31.37+7.27^{\Phi}$ \\
\hline
\end{tabular}

Data are expressed as mean \pm SE; $n=6$. For Student's t-test significant $\mathrm{p}$ value $\mathrm{Ah}, \mathrm{BZ}, \mathrm{BZ}+\mathrm{AhC}$, and $\mathrm{AhP}+\mathrm{BZ}$ versus control ${ }^{*} \mathrm{p} \leq 0.05,{ }^{* *} \mathrm{p} \leq 0.01$ and therapy versus $\mathrm{BZ}$ $\Phi \mathrm{p} \leq 0.05$ and $\Phi \Phi \mathrm{p} \leq 0.01$. Ah: Atriplex halimus, AhC: Atriplex halimus Curative treatment, AhP: Atriplex halimus Preventive treatment, BZ: Benzene, SE: Standard error

Table 4: Mean blood glucose levels and blood biochemical value in the control and experimental groups

\begin{tabular}{lllll}
\hline Parameters & Serum uric acid $(\mathrm{mg} / \mathbf{l})$ & Serum albumin $(\mathrm{g} / \mathbf{l})$ & Serum $\mathrm{LDH}(\mathrm{U} / \mathbf{l})$ & Serum \\
\hline Control & $19.67 \pm 1.33$ & $31.83 \pm 1.33$ & $3166 \pm 149$ & $72.33 \pm 2.47$ \\
Ah & $14.59 \pm 0.55^{* * *}$ & $39.33 \pm 1.41$ & $2227 \pm 135^{* *}$ & $83.83 \pm 3.42^{*}$ \\
BZ & $17.78+1.83$ & $31.17+1.17$ & $3324+216$ & $68.83+1.14^{*}$ \\
BZ+AhC & $18.74+1.18$ & $25.40+1.29$ & $3098+118^{\Phi}$ & $70+1.96$ \\
AhP+BZ & $12.00+0.56^{* * * \Phi \Phi}$ & $37.6+2.23$ & $2831+187^{\Phi}$ & $65.25+2.14^{*}$ \\
\hline
\end{tabular}

Data are expressed as mean \pm SE; $n=6$. For Student's t-test significant $\mathrm{p}$ value $\mathrm{Ah}, \mathrm{BZ}, \mathrm{BZ}+\mathrm{AhC}$, and $\mathrm{AhP}+\mathrm{BZ}$ versus control ${ }^{\mathrm{p}} \leq 0.05$, ${ }^{* *} \mathrm{p} \leq 0.01$ and ${ }^{* * *} \mathrm{p} \leq 0.001$ and therapy versus BZ $\Phi \mathrm{p} \leq 0.05$ and $\Phi \Phi \mathrm{p} \leq 0.01$. Ah: Atriplex halimus, AhC: Atriplex halimus Curative treatment, AhP: Atriplex halimus Preventive treatment, BZ: Benzene. LDH: lactate dehydrogenase, SE: Standard error

Table 5: Oxidative stress parameters in kidney of control and experimental groups

\begin{tabular}{llll}
\hline Parameters & MDA (nmol/mg prot) & GSH (nmol/g tissue) & GST (nmol/min/g tissue) \\
\hline Control & $3.78 \pm 0.33$ & $2.97 \pm 0.21$ & $5.96 \pm 0.32$ \\
Ah & $3.98 \pm 0.18$ & $2.42 \pm 0.31$ & $6.26 \pm 0.65$ \\
BZ & $4.52+0.065^{* *}$ & $2.28+0.051^{* *}$ & $4.40+0.20^{* *}$ \\
BZ+AhC & $5.26+0.085^{* * * \Phi \Phi}$ & $2.05+0.3^{*}$ & $5.62+0.42^{\Phi}$ \\
AhP+BZ & $3.49+0.19^{\Phi \Phi}$ & $2.66+0.15^{\Phi}$ & $6.31+0.044$ \\
\hline
\end{tabular}

Data are expressed as mean \pm SE; $n=6$. For Student's t-test significant $\mathrm{p}$ value Ah, BZ, BZ+AhC, and AhP+BZ versus control *p $\leq 0.05$, ** $\leq 0.01$ and ${ }^{* * *} \mathrm{p} \leq 0.001$ and therapy versus BZ $\Phi \mathrm{p} \leq 0.05$ and $\Phi \Phi \mathrm{p} \leq 0.01$. Ah: Atriplex halimus, AhC: Atriplex halimus Curative treatment, AhP: Atriplex halimus Preventive treatment, BZ: Benzene. MDA: Malondialdehyde, GSH: Reduced Glutathione, GST: Glutathione S transferase, CAT: Catalase, SE: Standard error 
for curative effect $(\mathrm{BZ}+\mathrm{AhC})$ and totally restored the MDA $(\mathrm{p}<0.01)$, GSH $(p<0.05)$ levels and CAT $(p<0.01)$, and GST $(p<0.05)$ activities for preventive effect $(\mathrm{AhP}+\mathrm{BZ})$. In addition, treatment with the plant extract alone does not cause any change in oxidative stress parameters.

\section{Correlations between analyzed parameters}

The results in Table 6 represent the correlation between serum kidney markers (creatinine and urea) and kidney oxidative stress markers (MDA, GSH, GST, and CAT) for experimental groups. Our results showed that there was a significant positive correlation $(p<0.05)$ between serum creatinine concentration and kidney MDA level. However, there was a significant negative correlation between serum creatinine concentration and kidney antioxidative markers GSH $(p<0.05)$, GST $(p<0.001)$, and CAT $(p<0.01)$. In addition, there was a significant positive correlation $(\mathrm{p}<0.05)$ between serum urea concentration and kidney MDA level and a negative correlation between serum urea concentration with GSH $(\mathrm{p}<0.01)$ level and GST $(\mathrm{p}<0.01)$ activity, but there was no significant correlation between serum urea and kidney CAT activity.

\section{Histopathological studies}

Microscopic observation of histological sections of kidney from the control and Ah treated rat showed normal renal parenchyma with glomeruli, tubules and normal cortical, and medullary areas (Fig. 2a and b). However, histological sections of the BZ exposed rat kidney revealed severe degeneration represented by necrosis and glomerular hypertrophy apparent to their architecture with tubular necrosis, dilatation, and tubular vacuolation. In addition, hemorrhage, foci of inflammation and large space of Bowman, was observed in the kidney sections of this group (Fig. 2c). Kidney section of (AhP+BZ) groups when rats received the aqueous extract of Atriplex protective treatment with BZ indicated protection from tubule and glomerulus (Fig. 2e). Atriplex curative treatment partially decreased severe damage to kidney tissue (Fig. 2d). The histopathological changes are graded and summarized in Table 7. Histological grading was made according to four severity grades: - (none), + (mild), ++ (moderate), and +++ (severe).

\section{DISCUSSION}

The finding of this study indicates that BZ-induced nephrotoxicity in the rat through the increase relative kidney weight, serum urea and creatinine levels, and alters renal antioxidant and oxidative stress parameters and also caused histopathological alterations in kidney tissue. However, our study indicated that $\mathrm{Ah}$ has a dramatically preventive effect on BZ-induced nephrotoxicity through the potential restoration of biochemical and histopathological parameters in kidney tissue rat. As well, in a curative mode, Ah partially restored the renal

Table 6: Correlation coefficients and the significant levels of different kidney marker components in rats

\begin{tabular}{llllll}
\hline Component vs. & \multicolumn{2}{l}{ Creatinine } & & & Urea \\
\cline { 2 - 3 } \cline { 6 - 6 } & $\mathbf{R}$ & $\mathbf{P}$ & & $\mathbf{R}$ & $\mathbf{P}$ \\
\hline Kidney MDA & 0.65 & 0.04 & & 0.88 & 0.001 \\
Kidney GSH & -0.75 & 0.012 & & -0.76 & 0.01 \\
Kidney GST & -0.9 & 0 & & -0.83 & 0.003 \\
Kidney CAT & -0.81 & 0.005 & & -0.52 & 0.111 \\
\hline
\end{tabular}

damage, suggesting that Atriplex exhibits inhibitory action thus causing nephroprotection.

BZ exposure at a dose $(100 \mathrm{mg} / \mathrm{kg})$ resulted in a significant decrease in body weight and increase in relative kidney weight. Ibrahim et al. reported a significant decline in the body weight gain of the animals intoxicated with BZ. Moreover, according to him; this reduced of the body weights may be due to the overall increased degradation of lipids and proteins as a result of the direct effects of BZ [26]. Then, cotreatment of Atriplex with BZ restored the weight of the body and kidney as normal, which can indicate the prevention against the injury effect of BZ on kidney as evidenced macroscopically.

The increased levels of urea and creatinine are considered as the marker of impaired kidney functions $[27,28]$. Thus, in our study, an increase of plasma urea and creatinine reflected the diagnosis of renal damage. These results were inconsistent with the study of Khan and Yusufi [29]. On other, occupational exposure to BZ in petrol stations was showed that serum creatinine was significantly higher in the exposed group than in the unexposed employees [30]. Aqueous extract of Ah preventive treatment decreased the raised serum urea and creatinine and maintained healthy kidney function, but low effect, we observed for curative treatment in these parameters. Moreover, BZ orally administrated to rats caused a significant decrease in serum protein concentration and less increase in serum LDH activity. However, BZ

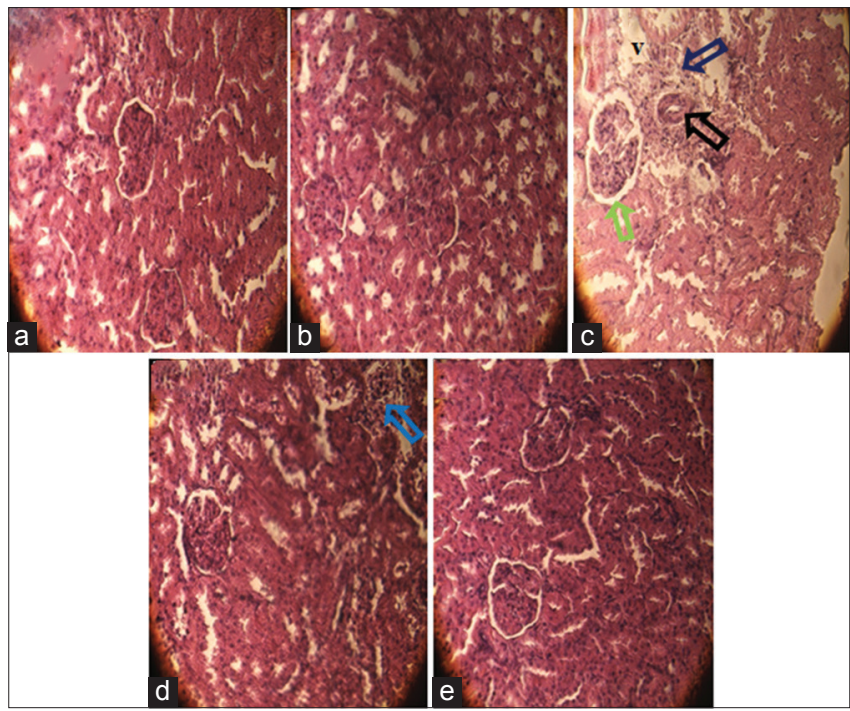

Fig. 2: (a) The histological examination of kidney from a control rat showing normal architecture $\times 400$. (b) Kidney section of Atriplex halimus treated rats showing a healthy renal architecture $\times 400$. (c) Benzene exposed rats kidney showing focus of inflammation (blue arrow), glomerular necrosis (black arrow), large space of bowman (green arrow), and vacuolation (V) $\times 400$. (d) A. halimus curative treated rat kidney showing a moderate degree of kidney damage and few inflammatory cell, protection from tubules, and glomerulus degradation $\times 400$. (e) Kidney sections of benzene rats prevented by $A$. halimus showed the normal appearance of tubules and glomerulus $\times 400$

Table 7: Semi-quantitative recording of architectural damage on histopathological analysis of the kidney of control and treated rats

\begin{tabular}{|c|c|c|c|c|c|}
\hline Parameters & $\begin{array}{l}\text { Dilatation and tabular } \\
\text { vacuolation }\end{array}$ & $\begin{array}{l}\text { Inflammatory } \\
\text { infiltration }\end{array}$ & $\begin{array}{l}\text { Tubular and glomerular } \\
\text { necrosis }\end{array}$ & $\begin{array}{l}\text { Large space of } \\
\text { Bowman }\end{array}$ & $\begin{array}{l}\text { Hypertrophy } \\
\text { glomerular }\end{array}$ \\
\hline Control & - & - & - & - & - \\
\hline $\mathrm{Ah}$ & - & - & - & - & - \\
\hline $\mathrm{BZ}$ & +++ & ++++ & ++++ & ++++ & +++ \\
\hline $\mathrm{BZ}+\mathrm{AhC}$ & + & ++ & ++ & + & + \\
\hline $\mathrm{AhP}+\mathrm{BZ}$ & - & - & - & + & - \\
\hline
\end{tabular}

Ah: Atriplex halimus, AhC: Atriplex halimus Curative treatment, AhP: Atriplex halimus Preventive treatment, BZ: Benzene 
exposure did not affect markedly serum glucose, uric acid, and albumin concentration.

Recent studies reported that BZ-initiated toxicity has been linked to the production of ROS [31]. More specifically BZ can be enzymatically bioactivated to reactive metabolites (catechol, benzoquinones, hydroquinone, and phenol), which can produce ROS, decreases antioxidant activity, and hence increases oxidative stress [32,33]. Kidney is one of the major tissue sites of BZ's metabolites and BZ's conjugated metabolites for phenol, catechol, and hydroquinone, phenyl sulfate and hydroquinone glucuronide, and muconic acid [34,35]. These compounds form covalent adducts with diverse macromolecules such as nucleic acids and proteins in the kidney [36].

MDA is one of the major lipid peroxidation products and is used as a marker of cell membrane destruction and tissue damage [37]. Our data demonstrated that BZ administration caused a significant increase in MDA levels as compared to control group in the kidney tissue, which reflects extensive lipid peroxidation process in the kidney of rats after BZ exposure. Our results are in good agreement with Çavuşoğlu et al. who found that BZ administration at a dose $50 \mathrm{mg} / \mathrm{kg}$ of body weight for 50 days caused a significant increase in kidney MDA [38].

Results from the present study demonstrate that the levels of endogenous antioxidants both enzymatic and non-enzymatic (GSH and CAT, GST) are significantly lowered in the BZ-treated group compared to the normal control rats, which are supported by the findings of Verma and Rana [39] who reported that BZ administration decreased the level of GSH in rats kidney. Furthermore, Hetal [40] demonstrated that BZ exposure leads to a decrease in antioxidant enzymes activity. The sulfhydryl reduced GSH is a small intracellular thiol (-SH) molecule, play an essential role in the detoxification processes [41]. Furthermore, previous studies indicated that GSH depletion is one of the important consequences of toxic injury; which might be consumed during the metabolic reaction of BZ [42]. CAT and GST antioxidant enzymes play a critical role in the protection of tissues from the deleterious effects of BZ. GST represent a major group of detoxifying enzymes [43], which catalyze the detoxification of $\mathrm{BZ}$ oxepin, metabolite potential reactive, and carcinogenic of BZ through conjugation with GSH and excretion in the urine as S-phenylmercapturic acid [44]. Finally, as an H2O2 scavenger, CAT has been shown to play an important role in the protection of cells against toxicity from the hydroquinone metabolite [45]. Therefore, the decreased activities of these antioxidant enzymes indicated a failure of the antioxidant defense system in BZ group, may have been due to oxidation reactions initiated by BZ metabolites and the influx of ROS induced by BZ exposure.

Extract Ah treatment, (especially preventive treatment) dramatically decrease MDA level and increase GSH, CAT, and GST level in the BZ group. This effect can be attributed to the activity of its antioxidants and the ROS scavenging capacity. In this study, aqueous extract of Ah contains higher levels of total phenolic and condensed tannin. Many researchers reported a significantly high correlation between the high antioxidant activity of plant extracts and its high total phenolic compounds. Further, according to the previous research, Ah contain a wide range of bioactive metabolites. It is a source of Vitamins A, C, and D, polyphenols, flavonoids, tannins, alkaloids, saponins, and resins [46]. These constituents are good indicators for the antioxidant activity of this plant that may be involved in the prevention of cellular damage and many human diseases, caused by free radicals $[47,48]$. These antioxidants molecules interact with free radicals and terminate the chain reaction before vital molecules are damaged. They donate an electron to stabilize a free radical [49]. Therefore, it may be suggested that Ah treatment can reduce the BZ toxicity by decreasing the oxidative stress through the activation of antioxidant defense systems, the ROS scavenging and the inhibition of LPO in BZ administered rats. These observations might indicate that Atriplex has protective effects on BZinduced oxidative stress and it may potentially reduce the unfavorable effects of BZ in the kidney.
Histopathological observation of renal tissue sections from the BZ group confirmed the biochemical results and showed clear signs of nephrotoxicity. Stained sections reveal they marked severe glomerular and tubular degeneration, glomeruli hypertrophy, loss of brush border membrane, vascular congestion, inflammatory cell infiltration, vacuolization of tubular cells, tubular dilatation, and increased number of pyknotic nuclei. Overproduction of ROS in chronic BZ exposure can cause damage of important biomolecules (proteins, lipids, and DNA) and increase intra-renal pro-inflammatory and fibrogenic factors, which can lead to cell death (necrotic) and chronic inflammation associated with tissue renal damage [50]. However, Atriplex improved the renal histological changes with normal glomeruli and tubules in preventive effect. This protective effect of the extract may be attributed to the presence of antioxidant and anti-inflammatory components in this plant.

\section{CONCLUSION}

The current study demonstrates that the administration of Ah can reduce (or completely prevent) several toxicities induced by BZ in kidneys. This protective potential may involve the powerful antioxidant properties of this plant by eliminating free radicals and oxidative damages caused by BZ. Hence, dietary supplementation with Ah could be a useful method to protect populations at high risk of environmental and/or occupational BZ chronic intoxication, and for scavenging the free radicals generated in the kidney.

\section{ACKNOWLEDGMENTS}

The first author would like to thank the Faculty of Sciences of Nature and Life, University of El Oued, Algeria, for the permission to utilize the institute facilities to make this work.

\section{AUTHOR'S CONTRIBUTIONS}

The conception of the study, drafting the article and interpretation of data was done by Khaoula Zeghib author; Djahra Ali Boutlelis author was responsible for the acquisition of data and analysis.

\section{CONFLICTS OF INTEREST}

The authors declare that they have no conflicts of interest.

\section{REFERENCES}

1. Sonawane B, Bayliss D. Toxicological Review of Benzene (Non cancer effects) (CAS No. 71-43-2). In: Support of Summary Information on the Integrated Risk Information System. Washington, DC, USA: U.S. Environmental Protection Agency; 1998.

2. Mathews JM, Etheridge AS, Matthews HB. Highlight dose-dependent metabolism of benzene in hamsters rats and mice. J Environ Sci 1998;44:14-21.

3. Çavuşoğlu K, Yalçin E, Yapar K, Gür B, Çiçek F. The protective role of grape seed extract against chronic toxicity of benzene in swiss albino mice. Cumhuriyet Sci J 2014;35:1-11.

4. Maltoni C, Ciliberti A, Cotti G, Conti B, Belpoggi F. Benzene, an experimental multipotential carcinogen: Results of the long-term bioassays performed at the bologna institute of oncology. Environ Health Perspect 1989;82:109-24.

5. Verma Y, Rana, SVS, Singh N. Simultaneous exposure to benzene and alcohol in petrol pump operators. Int J Pharm Sci Res 2016;7:2606-10.

6. Medinsky MA, Schlosser PM, Bond JA. Critical issues in benzene toxicity and metabolism: The effect of interactions with other organic chemicals on risk assessment. Environ Health Perspect 1994;102 Suppl 9:119-24.

7. Emara AM, El-Bahrawy H. Green tea attenuates benzene-induced oxidative stress in pump workers. J Immunotoxicol 2008;5:69-80.

8. Falzone L, Marconi A, Loreto C, Franco S, Spandidos DA, Libra M, et al. Occupational exposure to carcinogens: Benzene, pesticides and fibers (Review). Mol Med Rep 2016;14:4467-74.

9. El Batsh MM, Zakaria SS, Gaballah HH. Protective effects of alphalipoic acid against benzene induced toxicity in experimental rats. Eur Rev Med Pharmacol Sci 2015;19:2717-24.

10. Derouiche S, Zeghib K, Gharbi S, Khelef Y, Feriani A. Protective effects of aristolochia longa and aquilaria malaccensis against lead 
induced acute liver injury in rats. J Acute Dis 2017;6:193-19.

11. Tucker PS, Scanlan AT, Dalbo VJ. Chronic kidney disease influences multiple systems: Describing the relationship between oxidative stress, inflammation, kidney damage, and concomitant disease. Oxid Med Cell Longev 2015;2015:806358.

12. Oh GS, Kim HJ, Shen A, Lee SB, Khadka D, Pandit A, et al. Cisplatininduced kidney dysfunction and perspectives on improving treatment strategies. Electrolyte Blood Press 2014;12:55-65.

13. Afravy M, Angali K, Khodadadi A, Ahmadizadeh M. The protective effect of buffalo's milk against toluene induced-nephrotoxicity in rats. J Nephropathol 2017;6:174-9.

14. Basu S, Das M, Sen A, Datta G. Protective role of crude extract of Amorphophallus Campanulatus against ethanol-induced oxidative renal damage. Asian J Pharm Clin Res 2018;11:240-50.

15. Nedjimi B, Guit B, Toumi M, Beladel B,Akam A. Daoud Y.Atriplex halimus Subsp. schweinfurthii (Chenopodiaceae): Description, écologie et utilisations pastorales et thérapeutiques. Fourrages 2013;216:333-8.

16. Kabbash AM. Macroscopic and microscopic charachterization of Atriplex halimus L. growing in egypt and in vitro evaluation of its cytotoxic activity. W J Pharm Pharm Sci 2016;5:84-100.

17. Murugan R, Parimelazhagan T. Comparative evaluation of different extraction methods for antioxidant and anti-inflammatory properties from Osbeckia parvifolia Arn. An in vitro approach. J King Saud Univ Sci 2014;26:267-75.

18. Li HB, Cheng KW, Wong CC, Fan KW, Chen F, Jiang Y. Evaluation of antioxidant capacity and total phenolic content of different fractions of selected microalgae. Food Chem 2007;102:771-6.

19. Broadhurst RB, Jones WT. Analysis of condensed tannins using acidified vanillin. J Sci Food Agric 1978;29:788-94.

20. Snyder R, Hedli CC. An overview of benzene metabolism. Environ Health Perspect 1996;104 Suppl 6:1165-71.

21. Quintanilha AT, Packer L, Davies JM, Racanelli TL, Davies KJ. Membrane effects of vitamin E deficiency: Bioenergetic and surface charge density studies of skeletal muscle and liver mitochondria. Ann N Y Acad Sci 1982;393:32-47.

22. Weckbecker G, Cory JG. Ribonucleotide reductase activity and growth of glutathione-depleted mouse leukemia L1210 cells in vitro. Cancer Lett 1988;40:257-64.

23. Habig WH, Pabst MJ, Jakoby WB. Glutathione S-transferases. The first enzymatic step in mercapturic acid formation. J Biol Chem 1974;249:7130-9.

24. Aebi H. "Catalase in vitro". Methods Enzymol 1984;105:121-6.

25. Bradford MM. A rapid and sensitive method for the quantitation of microgram quantities of protein utilizing the principle of protein-dye binding. Anal Biochem 1976;72:248-54.

26. Ibrahim KS, Saleh ZA, Farrag AR, Shaban EE. Protective effects of zinc and selenium against benzene toxicity in rats. Toxicol Ind Health 2011;27:537-45.

27. Kadeche L, Bourogaa E, Saoudi M, Boumendjel A, Djeffal A, El Feki A, et al. Ameliorative effects of vanillin against metribuzin-induced oxidative stress and toxicity in rats. Int J Pharm Pharm Sci 2017;9:56-62.

28. Moirangthem RS, Gunindro N, Takhellambam DS, Khuraijam SD, Meena N, Rita S. Protective effect of Phyllanthus fraternus against cyclophosphamide induced nephrotoxicity in rats. Int J Basic Clin Pharmacol 2017;6:984-9.

29. Khan S, Yusufi AN. Effect of benzene on the enzymes of carbohydrate metabolism, brush border membrane (BBM) and oxidative stress in kidney and other rat tissues. Biol Med 2009;1:28-41.

30. Neghab M, Hosseinzadeh K, Hassanzadeh J. Early liver and kidney dysfunction associated with occupational exposure to sub-threshold limit value levels of benzene, toluene, and xylenes in unleaded petrol. Saf Health Work 2015;6:312-6.

31. Badham HJ, Renaud SJ, Wan J, Winn LM. Benzene-initiated oxidative stress: Effects on embryonic signaling pathways. Chem Biol Interact 2010;184:218-21.

32. Fenga C, Gangemi S, Teodoro M, Rapisarda V, Golokhvast K, Docea AO, et al 8-hydroxydeoxyguanosine as a biomarker of oxidative DNA damage in workers exposed to low-dose benzene. Toxicol Rep 2017:4:291-5

33. Uzma N, Kumar BS, Hazari MA. Exposure to benzene induces oxidative stress, alters the immune response and expression of p53 in gasoline filling workers. Am J Ind Med 2010;53:1264-70.

34. Wilbur S, Wohlers D, Paikoff S, Keith LS, Faroon O. ATSDR evaluation of health effects of benzene and relevance to public health. Toxicol Ind Health 2008;24:263-398.

35. Kalf GF. Recent advances in the metabolism and toxicity of benzene. Crit Rev Toxicol 1987;18:141-59.

36. Bayliss D, Jinot J, Sonawane B. Toxicological Review of Benzene (CAS No. 71-43-2). In Support of Summary Information on the Integrated Risk Information System; EPA/635/R-02/001F. Washington, DC, USA: U.S. Environmental Protection Agency; 2002

37. Derouiche S, Rezzag MO, Serouti A. Triazinone herbicide metribuzin induced acute liver injury: A study of animal model. J Acute Dis 2018;7:152-7

38. Çavuşoğlu K, Yalçin E, Oruç E, Gür B, Çiçek F. The effects of grape seed extract against toxicity of benzene on liver and kidney tissues of albino mice: Biochemical evaluation. Turk J Biochem 2015;40:66-73.

39. Verma Y, Rana SV. Modulation of CYP4502E1 and oxidative stress by testosterone in liver and kidney of benzene treated rats. Indian J Exp Biol 2008;46:568-72.

40. Hetal R. Evaluation of benzene induced histopathological alteration in rat. Int J Adv Res 2016;4:563-7.

41. Sairazi SM, Sirajudeen KN, Muzaimi M, Swamy M, Asari MA, Sulaiman SA. Tualang honey attenuates kainic acid-induced oxidative stress in rat cerebellum and brainstem. Int J Pharm Pharm Sci 2017;9:155-62.

42. Abd-Elshakour A, El-Ebiarie AS, Ibrahim YH, Abd-Elmoneim AE, ElMekawy AM. Effect of benzene on oxidative stress and the functions of liver and kidney in rats. J Environ Occup Sci 2015;4:34-9.

43. Elsayed AS. Hematotoxicity and oxidative stress caused by benzene. Pyrex J Biomed Res 2015;1:74-80.

44. Zarth AT, Murphy SE, Hecht SS. Benzene oxide is a substrate for glutathione S-transferases. Chem Biol Interact 2015;242:390-5.

45. Ahmad S, Agrawal R, Agrawal DK, Rao GS. Bioreactivity of glutathionyl hydroquinone with implications to benzene toxicity. Toxicology 2000;150:31-9.

46. Chikhi I, Allali H, Dib MA, Medjdoub H, Tabti B. Antidiabetic activity of aqueous leaf extract of Atriplex halimus L. (Chenopodiaceae) in streptozotocin-induced diabetic rats. Asian Pac J Trop Dis 2014;4:181-4.

47. Kaouachi A, Derouiche S. Phytochemical analysis, DPPH antioxidant activity and Acute toxicity of bark aqueous extracts of Pinus halepensis. Res J Chem Env Sci 2018;6:86-91.

48. Mohammedi Z. Resistance, pharmacology properties and nutritional value of a shrub from arid environments Atriplex halimus. Res J Med Plant 2016;10:10-8.

49. Mohammadi A, Ahmadizadeh M. Effects of antioxidants on xenobiotics-induced nephrotoxicity. J Renal Inj Prev 2018;7:56-7.

50. Cottone S, Lorito MC, Riccobene R, Nardi E, Mulè G, Buscemi S, et al. Oxidative stress, inflammation and cardiovascular disease in chronic renal failure. J Nephrol 2008;21:175-9. 